\title{
Using the Secondary Pathway of Hemostasis in Endodontics for Fully Bone Healing: Report of Two Clinical Cases
}

\author{
Gustavo Alberto Obando-Pereda* \\ Odontología de la Universidad Católica de Santa María, Perú, South America \\ Received: December 19, 2017; Published: January 03, 2018
}

*Corresponding author: Gustavo Obando Pereda, Professor of Periodontics and Dental Implantology, Universidad Católica de Santa María, Urbanización San José s/n, Umacollo, Arequipa, Perú, South America, Tel: 5154 380038; Email: gobando@ucsm.edu.pe

\begin{abstract}
Chronic periapical lesion produce bone defect around apical zone of the accomplish teeth. The standard therapy follow an osteotomy, apical removing and profuse curettage to aim the remove infected tissue and granulomatous tissue. However, this procedure let carry, in many time, that the bone defect do not present any filler to rapid bone healing. Platelet rich in fibrin perform a rapid bone healing when is used to fill bone defects.
\end{abstract}

Keywords: Fibrin; Apicoectomy; Periapical disease

\section{Introduction}

Root canal treatment mains to eliminate all microorganisms into the canal complex to prevent any reinfection [1]. In many ways, this procedure does not eliminate all microorganisms from the root canal and the reinfection occurs producing and chronic inflammation than produce bone disorganization around the root. This bone destruction is known as apical lesion [1], where in radiographic examination appears this bone destruction as radiolucency around the root [2]. Dental clinicians treat this periapical lesion performing an apicoeptomy who mains retire the apical section of the compromise root, debridement and curettage of compromise tissue [2]. In fact, large bone defect is present after the procedure and bone substitutes are using to fill the defect. Many of these bone substitutes are particle commercial bone, commercial hydroxyapatite that only have an osteoconduction properties, delaying the healing [3]. The only material who gives an osteoconduction, osteoinduction and osteogenics properties is the autologous bone [4]. However, employed this bone, means a large surgery to pull a donor from chin, mandibular oblique line and other donor sites, provoking pain, stress and delayed surgery for the patient [5]. In this way platelet rich in fibrin (PRF) is an optimal autologous biological matrix that offer growing factors to be present in a wound healing provoking a rapid bone regeneration and repair [6].

\section{Clinical Cases}

\section{Clinical Case \# 1}

a. Periapical Lesion: A 25 years old female present a radiolucency zone at the upper right lateral central incisor compatible with a periapical lesion (Figure 1A).

b. Presurgical Therapy: the surgical procedure was explained to the patient and the informed consent was obtained. Pharmacology preparation of the patient included ketorolac (90mg), dexamethasone (4mg) and antibiotic prophylaxis with amoxicillin $(2 \mathrm{~g})$ one hour before the procedure.

c. PRF Preparation: the protocol was performing as described by Obando-Pereda: $10 \mathrm{~mL}$ of blood was drawn in $15 \mathrm{~mL}$ test tube without an anticoagulant and centrifuged immediately using a tabletop centrifuge (Greetmed Centrifuge mod. GT119$100 \mathrm{~T}$, China) at $3000 \mathrm{rpm}$ for 10 minutes. In absence of an anticoagulant, blood begins to coagulate immediately when is exposed to glass surface of test tube. Therefore, for successful preparation of PRF, blood must been collected and immediately centrifugation leads the clotting cascade initiated6. PRF was obtaining in the form of gelatinous plasma (Figures 1B \& 1C) [6]. 
d. Surgical Procedure: after proper isolation of the surgical field, the operative site was anaesthetized using $72 \mathrm{mg}$ of lidocaine with epinephrine (1:80000). An on line incision was made along the alveolar ridge soft tissue to retail a mucoperiostial flap. An osteotomy was conducted to reach the apical portion of teeth; subsequently apical portion was removed, the lesion was curettage to remove any granulose and infected tissue (Figure 1B). Immediately to curettage, the PRF was inserted in the bone defect. An autologous barrier was produced from PRF and this was putted on bone closed the osteotomy (Figure 1D). Soft tissue was approached and sutured to enhance a first intention wound healing. A periapical x-ray was performed immediately ending the surgical procedure (Figure 1E) and at 30 days (Figure $1 \mathrm{~F}$ ).

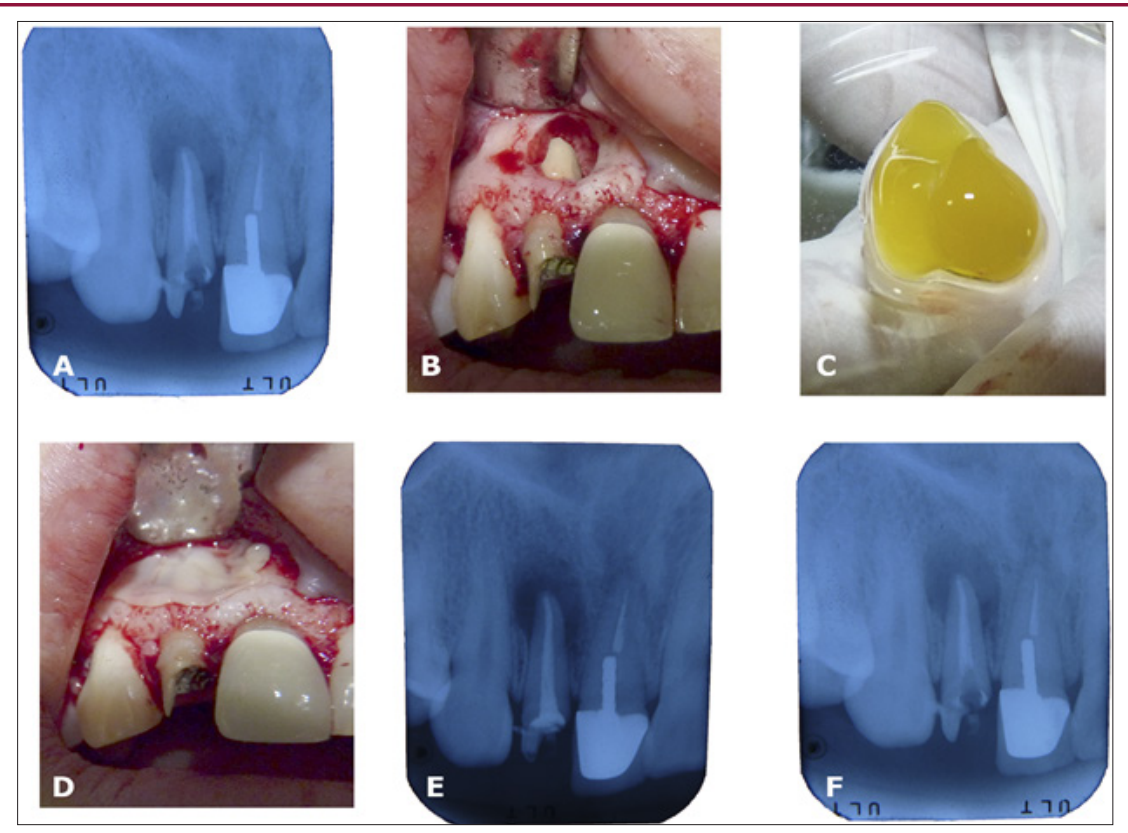

Figure 1: A. Periapical rx of upper right lateral incisor showed a periapical lesion; B. Osteotomy and visualization of apical portion and apicoceptomy; C. PRF clot obtained and divided in two parts; D. One part is entirely put in the bone defect; the other part was performed an autologous barrier; E. Periapical rx ending the surgery; F. Periapical rx at 30 days of surgery showed a bone matrix.

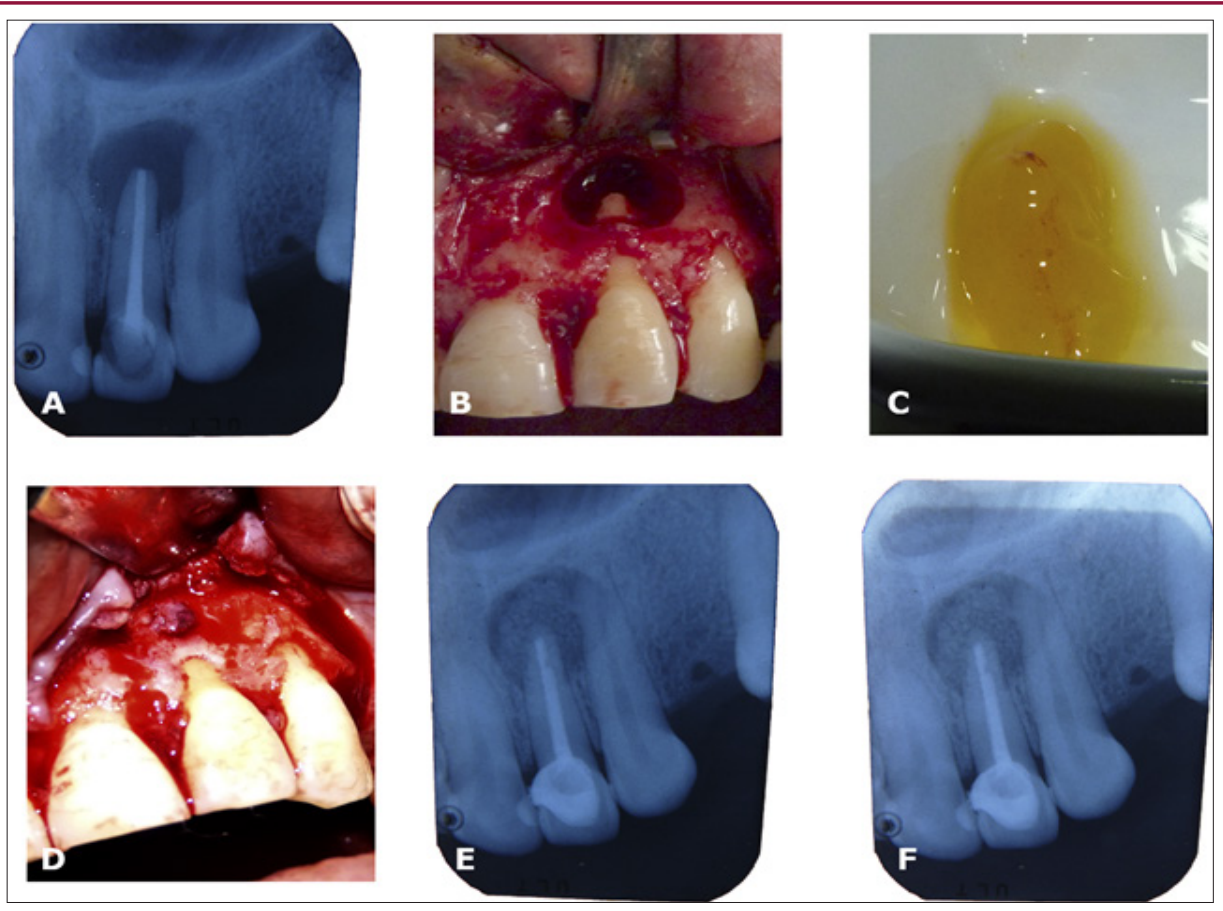

Figure 2: A. Periapical rx of upper right lateral incisor showed a periapical lesion; B. Osteotomy and visualization of apical portion and apicoceptomy; C. PRF clot obtained and divided in two parts; D. One part is entirely put in the bone defect, the other part was performed an autologous barrier; E. Periapical rx ending the surgery; F. Periapical rx at 30 days of surgery showed a bone matrix. 
e. Postoperative Healing: there was not post-operative complication and healing was satisfactory. Radiographic control suggested the formation of an osseous matrix in bone defect at 30 days.

\section{Clinical case \#2}

a. Periapical Lesion: A 20 years old female present a radiolucency zone at the upper left lateral central incisor compatible with a periapical lesion (Figure 2A).

b. Presurgical Therapy: the surgical procedure was explained to the patient and the informed consent obtained. Pharmacology preparation of the patient included ketorolac (90mg), dexamethasone (4mg) and antibiotic prophylaxis with amoxicillin $(2 \mathrm{~g})$ one hour before the procedure.

c. Surgical Procedure: after proper isolation of the surgical field, the operative site was anaesthetized using $72 \mathrm{mg}$ of lidocaine with epinephrine (1:80000). An on line incision was made along the alveolar ridge soft tissue to retail a mucoperiostial flap. An osteotomy was conducted to reach the apical portion of teeth; subsequently apical portion was removed, the lesion was curettage to remove any granulose and infected tissue (Figure 2B). Immediately to curettage, the PRF (Figure 2C) was inserted in the bone defect. An autologous barrier was produced from PRF and this was putted on bone closed the osteotomy (Figure 2D). Soft tissue was approached and sutured to enhance a first intention wound healing. A periapical $\mathrm{x}$-ray was performed immediately ending the surgical procedure (Figure 2E) and at 30 days (Figure 2F).

d. Postoperative Healing: there was not post-operative complication and healing was satisfactory. Radiographic control suggested the formation of an osseous matrix in bone defect at 30 days.

\section{Discussion}

The PRF protocol is frequently used with very favourable results for growth of hard and soft in the specialist dental clinic tissues [6]. In endodontic therapies the PRF protocol is not a frequently used, indeed, is used as a revascularization therapy with controversial results [7]. However, in many dental surgeries the PRF protocol has been showed a great results for bone regeneration in small a large bone defects alone [7] or combined with other materials [3]. In these clinical reports, the periapical lesion therapy was consist in the apical and infected tissue removal with the appropriated curettage. In this fact, a bone defect was done. PRF shows the benefits of rapid bone regeneration in bone defects [6]. This observation is in accordance with Nagaveni [8]. This event can be explained by the realising of growth factors mainly and cytokines immersed in the fibrin clot that can control the inflammatory response and the regenerative properties of immune system to modulate of cellular migration and proliferation, accelerating the bone healing [6].

The rapid bone regeneration is directly related the biological growing factor as BMPs. Some studies showed a rapid bone regeneration when BMPs are using to fill bone defects [9]. However, commercial BMPs are very expensive and the cares of these proteins are many to avoid its denaturation. PRF offers similarities results because presents many growing factors including pre-BMPs which promotes acceleration of bone healing in bone defects treatments shortening the bone healing time [6]. Indeed, PRF protocol can be used for regenerate small and large bone defect produced by periapical lesion with predictable clinical results.

\section{References}

1. Del Fabbro M, Corbella S, Sequeira-Byron P, Tsesis I, Rosen E, et al. (2016) Endodontic procedures for retreatment of periapical lesions. Cochrane Database Syst Rev 10: CD005511.

2. Del Fabbro M, Samaranayake LP, Lolato A, Weinstein T, Taschieri S (2014) Analysis of the secondary endodontic lesions focusing on the extraradicular microorganisms: an overview. J Investig Clin Dent 5(4): 245-254.

3. Vaishnavi C, Mohan B, Narayanan LL (2011) Treatment of endodontically induced periapical lesions using hydroxyapatite, platelet-rich plasma, and a combination of both: An in vivo study. J Conserv Dent 14(2): 140146.

4. Albrektsson T, Johansson C (2001) Osteoinduction, osteoconduction and osseointegration. Eur Spine J 10(Suppl 2): S96-101.

5. Zizzari VL, Zara S, Tete G, Vinci R, Gherlone E, et al. (2016) Biologic and clinical aspects of integration of different bone substitutes in oral surgery: a literature review. Oral Surg Oral Med Oral Pathol Oral Radiol 122(4): 392-402.

6. Obando-Pereda G (2016) Autologus barriers, fillers and growing factors: using the secondary pathway of hemostasis in dental clinics - report of four clinical cases. Open Access Journal of Dental Sciences.

7. Jadhav GR, Shah D, Raghvendra SS (2015) Autologus Platelet Rich Fibrin aided Revascularization of an immature, non-vital permanent tooth with apical periodontitis: A case report. J Nat Sci Biol Med 6(1): 224-225.

8. Nagaveni NB, Kumari KN, Poornima P, Reddy V (2015) Management of an endo-perio lesion in an immature tooth using autologous plateletrich fibrin: a case report. J Indian Soc Pedod Prev Dent 33: 69-73.

9. Herford AS, Boyne PJ (2008) Reconstruction of mandibular continuity defects with bone morphogenetic protein-2 (rhBMP-2). J Oral Maxillofac Surg 66(4): 616-624.

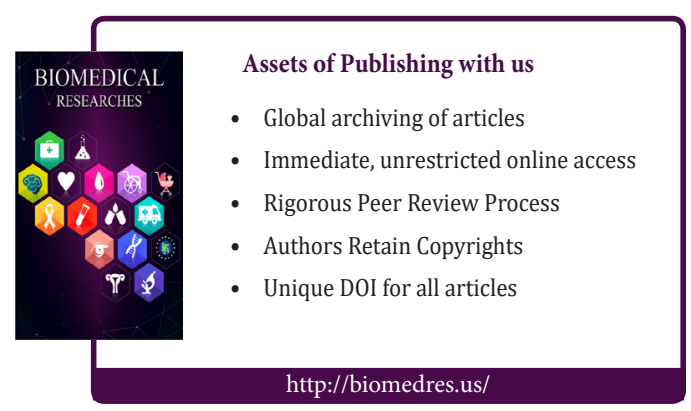

